\title{
Síndrome de Twiddler: Causa Rara de Disfunção de Pacemaker Definitivo
}

\author{
Twiddler Syndrome: Rare Cause of Definitive Pacemaker Dysfunction
}

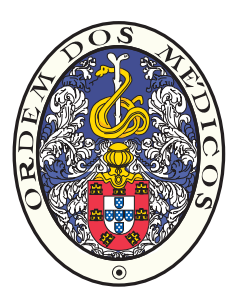

Davide MOREIRA ${ }^{1}$, Luís Ferreira dos SANTOS ${ }^{1}$, António COSTA ${ }^{1}$, Luís NUNES ${ }^{1}$, Jorge Oliveira SANTOS Acta Med Port 2014 Sep-Oct;27(5):652-654

\section{RESUMO}

Os autores apresentam um caso de síndrome de Twiddler, uma complicação rara após implantação de pacemaker definitivo, primeiramente descrito em 1968, complementado com uma abordagem à etiologia e manifestações desta entidade.

Palavras-chave: Síndrome de Twiddler; Pacemaker/efeitos adversos; Falha de Equipamento.

\section{ABSTRACT}

The authors present a case of Twiddler's syndrome, a rare complication after pacemaker implantation, first described in 1968. The article is complemented by an approach to the etiology and manifestations of this entity.

Keywords: Twiddler Syndrome; Pacemaker, Artificial/adverse effects; Equipment Failure.

\section{INTRODUÇÃO}

A síndrome de Twiddler é uma complicação conhecida após a implantação de pacemaker definitivo; foi descrito pela primeira vez por Bayliss et al após um doente ter manipulado e rodado o gerador na loca cirúrgica que resultou no desalojamento do electrocateter com consequente perda de captura. ${ }^{1}$

Os autores descrevem um caso clínico de síndrome de Twiddler diagnosticado vinte dias após a implantação de pacemaker definitivo.

\section{CASO CLÍNICO}

Homem de 74 anos, com queixas de tonturas paroxísticas, foi referenciado para avaliação por Cardiologista. No estudo efectuado apresentou em registo Holter de 24 horas bloqueio aurículo-ventricular 2:1 intermitente, sinto- mático, pelo que foi decidida a implantação de pacemaker definitivo de dupla-câmara. Foi implantado gerador Sensia L SEDRL1 $^{\odot}$, Medtronic ${ }^{\odot}$, EUA na região infra-clavicular esquerda e pela via cefálica esquerda foi posicionado eléctrodo ventricular no ápice do ventrículo direito e pelo mesmo acesso foi posicionado eléctrodo auricular no apêndice auricular direito tendo ambos sido implantados com modo de fixação passiva. Os testes de limiar agudo e sensing realizados peri-procedimento assim como a radiografia de tórax de duas incidências executada 24 horas após intervenção cirúrgica confirmaram a correcta posição e funcionamento do sistema anti-bradicardia (Fig. 1).

Vinte dias após a data da implantação o doente recorreu ao Serviço de Urgência por queixas de palpitações e soluços com dez dias de evolução. Na anamnese o doente

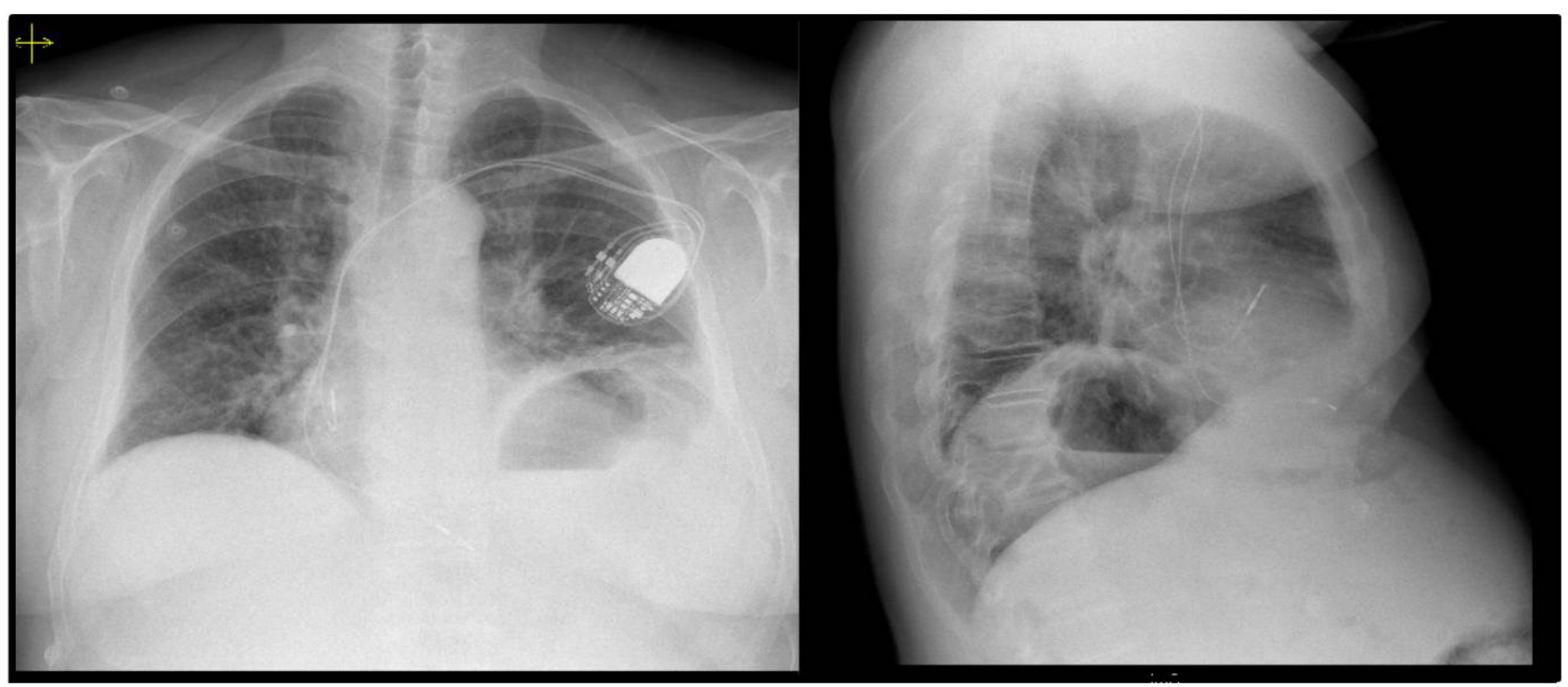

Figura 1 - Radiografia de tórax póstero-anterior e incidência oblíqua após implantação de pacemaker definitivo

1. Serviço de Cardiologia. Centro Hospitalar Tondela-Viseu. Viseu. Portugal.

Recebido: 29 de Setembro de 2013 - Aceite: 26 de Dezembro de 2013 | Copyright $\odot$ Ordem dos Médicos 2014 
negou manipulação consciente do gerador e ao exame objectivo era notável a ocorrência de eructações paroxísticas. Realizou ECG de 12 derivações que mostrou disfunção de sistema de pacemaker com falhas de sensing e de captura (Fig. 2) e radiografia de tórax que mostrou o desalojamento dos electrocateteres que se encontravam enrolados em torno do gerador (Fig. 3).

Esta situação, caracterizada por rotação do gerador, desalojamento dos electrocateteres e perda de captura com eventual estimulação diafragmática constitui, por definição, síndrome de Twiddler. A programação do modo de pacing para VVI com frequência cardíaca mínima de 30/ min permitiu a cessação dos sintomas referidos, tendo o doente permanecido internado em ritmo próprio com condução AV 1:1.

No dia seguinte procedeu-se à revisão cirúrgica com reposicionamento dos electrocateteres, não tendo sido verificada a existência de alteração da integridade destes; foi reforçada a posição do gerador sobre o músculo peitoral e o teste dos parâmetros permitiu confirmar o sucesso peri-procedimento. A radiografia de tórax conferiu a sua colocação correcta (Fig. 4).

No dia seguinte o doente teve alta clínica para o domicílio, permanecendo sem quaisquer intercorrências.

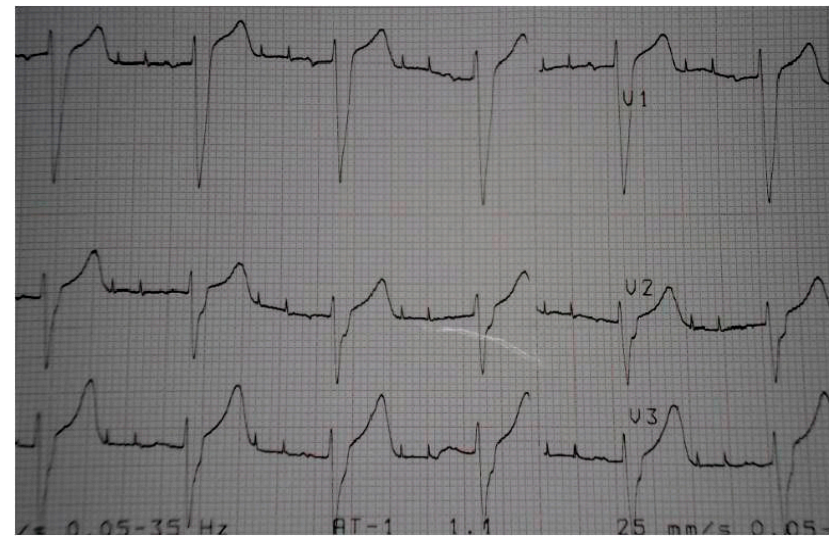

Figura 2 - Falhas de sensing e de captura visíveis no ECG de 12 derivações, com especial incidência nas derivações ântero-septais

\section{DISCUSSÃO}

Por síndrome de Twiddler entende-se o mau funcionamento permanente de pacemaker definitivo devido à manipulação do gerador pelo doente; ${ }^{2-4}$ esta síndrome foi primeiramente descrito em $1968^{5}$ e pode ocorrer com outros dispositivos intra-cardíacos como os cardio-desfibrilhadores implantáveis. ${ }^{6,7}$

A sequência de sintomas inicia-se com a manipulação consciente ou inconsciente do gerador, com subsequente desalojamento dos electrocateteres e cessação do pacing ventricular; o enrolamento dos electrocateteres em torno do gerador leva à estimulação do nervo frénico ipsilateral, com consequente pacing diafragmático e sensação de eructações. A progressão do enrolamento leva à estimulação do plexo braquial, com consequente movimentação rítmica dos membros superiores. .,3 $^{2,}$ Neste caso o doente negou manipulação consciente do gerador - o que acontece na maioria dos casos descritos ${ }^{6}$ - mas referiu que não cumpriu

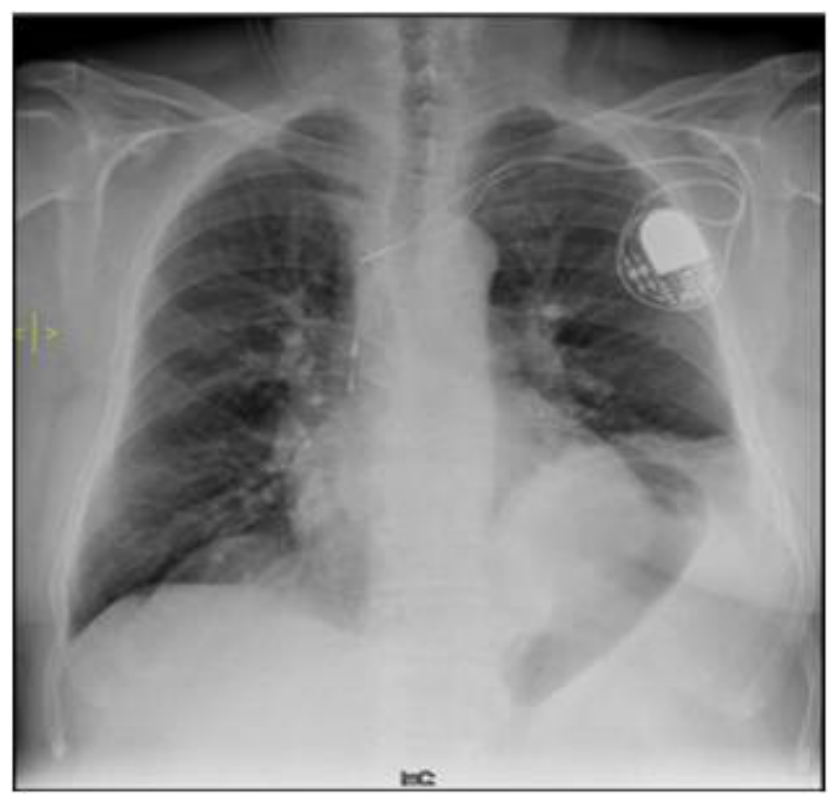

Figura 3 - Radiografia de tórax póstero-anterior; é notável o enrolamento dos electrocateteres em torno do gerador e o desalojamento dos electrocateteres

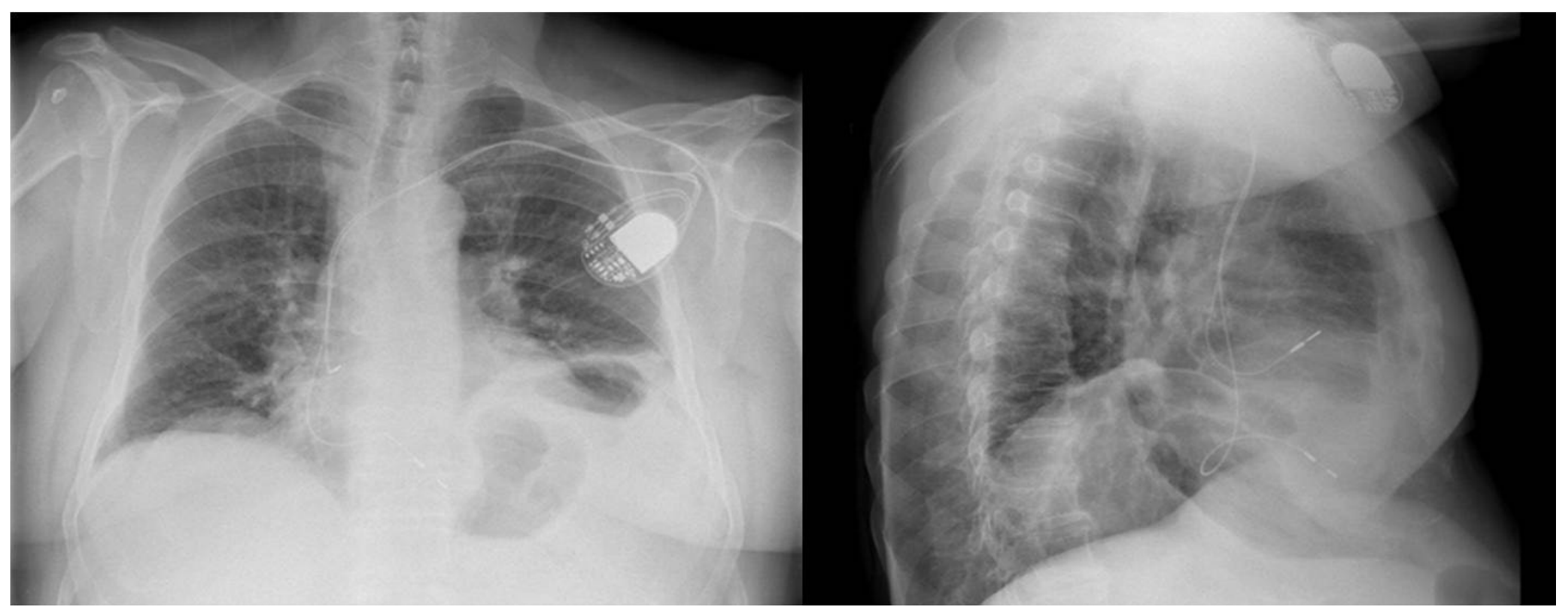

Figura 4 - Radiografia de tórax póstero-anterior após recolocação dos electrocateteres 
cuidadosamente as instruções de evitamento de movimentação do membro superior esquerdo o que deve ter contribuído para a ocorrência desta situação. ${ }^{6}$

A incidência deste síndrome varia entre $0,07 \%$ e $7 \%, 1,6$ e os factores de risco incluem idade avançada e obesidade - devido à maior lassidão do tecido celular subcutâneo - género feminino, patologia psiquiátrica e a pequenas dimensões do gerador relativamente à sua loca de colocação. ${ }^{1-3,6}$ A maioria dos casos ocorre no primeiro ano após a implantação do sistema de pacemaker, mas está descrita a ocorrência após este período. ${ }^{8}$ O diagnóstico é essencialmente radiológico e confirma-se através da exploração cirúrgica da loca de pacemaker. ${ }^{4}$

\section{REFERÊNCIAS}

1. Fahraeus T, Hoijer CJ. Early pacemaker twiddler syndrome. Europace. 2003;5:279-81.

2. Nicholson WJ, Tuohy KA, Tilkemeier P. Twiddler's Syndrome. N Engl J Med. 2003:348:1726-7.

3. Benezet-Mazuecos J, Benezet J, Ortega-Carnicer J. Pacemaker twiddler syndrome. Eur Heart J. 2007;28:2000.

4. Salazar-Ramirez C, Carballo-Ruiz M, Martos Rodriguez C, Garcia-Alcantara A. Twiddler syndrome as cause of loss of ventricular capture in a patient with definitive pacemaker. Med Intensiva. 2011;35:64.

5. Bayliss CE, Beanlands DS, Baird RJ. The pacemaker-twiddler's syn-

\section{CONCLUSÕES}

A minimização das dimensões da loca do gerador, a utilização de eléctrodos de fixação activa, o reforço da fixação do gerador mediante a realização de sutura muscular ou a utilização de bolsa de Parsonett podem minimizar a probabilidade de ocorrência ou de recorrência deste síndrome..$^{3,6}$

$\mathrm{O}$ interesse deste caso reside na raridade da sua ocorrência e na peculiaridade dos sintomas de apresentação.

\section{CONFLITOS DE INTERESSE}

Os autores declaram não terem quaisquer conflitos de interesse.

\section{FONTES DE FINANCIAMENTO}

Os autores declaram que o presente trabalho não foi objecto de qualquer financiamento.

drome: a new complication of implantable transvenous pacemakers. Can Med Assoc J. 1968;99:371-3.

6. Mandal S, Pande A, Kahali D. A rare case of very early pacemaker twiddler's syndrome. Heart Views. 2012;13:114-5.

7. Sharifi M, Inbar S, Neckels B, Shook H. Twiddling to the extreme: development of twiddler syndrome in an implanted cardioverter-defibrillator. J Invasive Cardiol. 2005;17:195-6.

8. Dursun I, Yesildag O, Soylu K, Yilmaz O, Yasar E, Meric M. Late pacemaker twiddler syndrome. Clin Res Cardiol. 2006;95:547-49. 


\section{Síndrome de Twiddler: Causa Rara de Disfunção de Pacemaker Definitivo}

Acta Med Port 2014:27:652-654

Publicado pela Acta Médica Portuguesa, a Revista Científica da Ordem dos Médicos

Av. Almirante Gago Coutinho, 151

1749-084 Lisboa, Portugal.

Tel: +351218428215

E-mail: submissao@actamedicaportuguesa.com

www.actamedicaportuguesa.com

ISSN:0870-399X | e-ISSN: 1646-0758

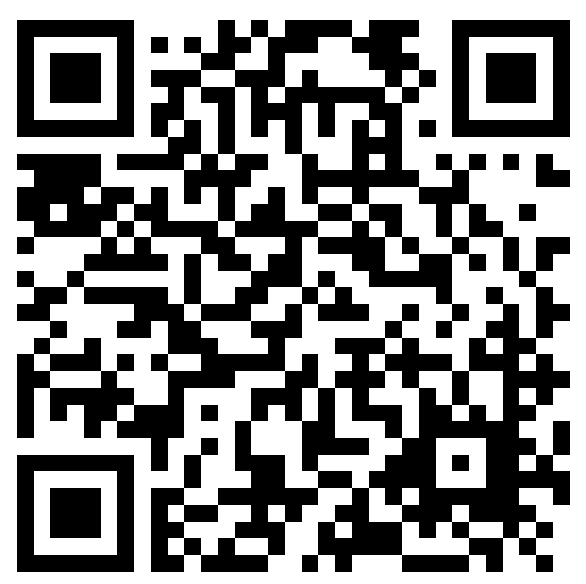

